SLAC-PUB-14208

Jlab-PHY-10-1135

July 2010

\title{
The AdS/QCD Correspondence and Exclusive Processes
}

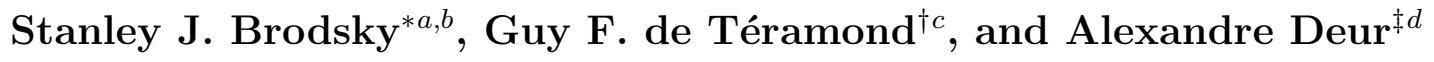 \\ ${ }^{a}$ SLAC National Accelerator Laboratory, Stanford University, Stanford, CA 94309, USA \\ ${ }^{b} \mathrm{CP}^{3}$-Origins, Southern Denmark University, Odense, Denmark \\ ${ }^{c}$ Universidad de Costa Rica, San José, Costa Rica \\ ${ }^{d}$ Thomas Jefferson National Accelerator Facility, Newport News, VA 23606, USA
}

\begin{abstract}
The AdS/CFT correspondence between theories in AdS space and conformal field theories in physical space-time provides an analytic, semi-classical, colorconfining model for strongly-coupled QCD. The soft-wall AdS/QCD model modified by a positive-sign dilaton metric leads to a remarkable one-parameter description of nonperturbative hadron dynamics at zero quark mass, including a zero-mass pion and a Regge spectrum of linear trajectories with the same slope in orbital angular momentum $L$ and radial quantum number $n$ for both mesons and baryons. One also predicts the form of the non-perturbative effective coupling $\alpha_{s}^{A d S}(Q)$ and its $\beta$-function which agrees with the effective coupling $\alpha_{g_{1}}$ extracted from the Bjorken sum rule. Light-front holography, which connects the fifth-dimensional coordinate of AdS space $z$ to an invariant impact separation variable $\zeta$, allows one to compute the analytic form of the frame-independent light-front wavefunctions, the fundamental entities which encode hadron properties as well as decay constants, form factors, deeply virtual Compton scattering, exclusive heavy hadron decays and other exclusive scattering amplitudes. One thus obtains a relativistic description of hadrons in QCD at the amplitude level with dimensional counting for hard exclusive reactions at high momentum transfer. As specific examples we discuss the behavior of the pion and nucleon form factors in the space-like and time-like regions. We also review the phenomenology of exclusive processes including some anomalous empirical results.
\end{abstract}

\footnotetext{
*Electronic address: sjbth@slac.stanford.edu

$\dagger$ Electronic address: gdt@asterix.crnet.cr

${ }^{\ddagger}$ Electronic address: deurpam@jlab.org
} 


\section{Introduction}

Exclusive processes play a key role in quantum chromodynamics, testing the primary quark and gluon interactions of QCD and the structure of hadrons at the amplitude level. Two basic pictures have emerged based on perturbative QCD (pQCD) and nonperturbative AdS/QCD. In pQCD hard scattering amplitudes at a high scale $Q^{2}>>\Lambda_{\mathrm{QCD}}^{2}$ factorize as a convolution of gauge-invariant hadron distribution amplitudes $\phi_{H}\left(x_{i}, Q\right)$ with the underlying hard scattering quark-gluon subprocess amplitude $T_{H}$. The leading power fall-off of the hard scattering amplitude follows from the conformal scaling of the underlying hard-scattering amplitude: $T_{H} \sim 1 / Q^{n-4}$, where $n$ is the total number of fields (quarks, leptons, or gauge fields) participating in the hard scattering [1, 2]. Thus the reaction is dominated by subprocesses and Fock states involving the minimum number of interacting fields. In the case of $2 \rightarrow 2$ scattering processes, this implies the dimensional counting rules $d \sigma / d t(A B \rightarrow C D)=F_{A B \rightarrow C D}(t / s) / s^{n-2}$, where $n=N_{A}+N_{B}+N_{C}+N_{D}$ and $N_{H}$ is the minimum number of constituents of $H$. The result is modified by the ERBL evolution [3, 4] of the distribution amplitudes and the running of the QCD coupling.

It is striking that the dimensional counting rules are also a key feature of nonperturbative AdS/QCD models [5]. Although the mechanisms are different, both the pQCD and AdS/QCD approaches depend on the leading twist interpolating operators of the hadron and their structure at short distances. In both theories, hadronic form factors at high $Q^{2}$ are dominated by the wavefunctions at small impact separation. This in turn leads to the color transparency phenomena [6, 7]. For example, measurements of pion photoproduction are consistent with dimensional counting $s^{7} d \sigma / d t\left(\gamma p \rightarrow \pi^{+} n\right) \sim$ constant at fixed CM angle for $s>7 \mathrm{GeV}$. The angular distributions seen in hard large $\mathrm{CM}$ angle scattering reactions are consistent with quark interchange [8], a result also predicted by the hard wall AdS/QCD model. Reviews are given in Refs. 9] and [10]. One sees the onset of perturbative QCD scaling behavior even for exclusive nuclear amplitudes such as deuteron photodisintegration (Here $n=1+6+3+3=13)$ and $s^{11} d \sigma / d t(\gamma d \rightarrow p n) \sim$ constant at fixed CM angle [11, 12, 13]. The measured deuteron form factor [14] also appears to follow the leading-twist QCD predictions [15] at large momentum transfers in the few GeV region. The six color-triplet quarks of the valence Fock state of the deuteron can be arranged as a sum of five different color-singlet states, only one of which can be identified with the neutron-proton state and can account for the large magnitude of the deuteron form factor at high scales. A measurement of $d \sigma / d t\left(\gamma d \rightarrow \Delta^{++} \Delta\right)$ in the scaling region can establish the role of "hidden-color" degrees of freedom [16] of 
the nuclear wavefunction in hard deuteron reactions.

In the case of pQCD, the near-constancy of the effective QCD coupling at small scales helps explain the general empirical success of the dimensional counting rules for the near-conformal power law fall-off of form factors and fixed-angle scaling [17].

Color transparency [6, 7] is a key property of color gauge theory, and it thus stands at the foundations of QCD. Color transparency has been confirmed in diffractive dijet production [18], pion photoproduction [19] and vector meson electroproduction [20], but it is very important to also systematically validate it in large-angle hadron scattering processes. Color transparency and higher-twist subprocesses [21, 22, 23, 24, 25] where the trigger hadron is produced directly, such as $u u \rightarrow p \bar{d}$, can account for the anomalous growth of the baryon-to-meson ratio with increasing centrality observed in heavy ion collisions at RHIC [26].

\section{Anomalies in Exclusive Processes}

Some exceptions to the general success of dimensional counting are known:

The transition form factor $F\left(Q^{2}\right)_{\gamma \rightarrow \pi^{0}}$ between a real photon and a pion has been recently measured at BaBar to high $Q^{2} \simeq 10 \mathrm{GeV}^{2}$, falling at high photon virtuality roughly as $1 / Q$ rather than the predicted $1 / Q^{2}$ fall-off. In contrast, preliminary measurements from BaBar [27] indicate that the transition form factors $F\left(Q^{2}\right)_{\gamma \rightarrow \eta}$ and $F\left(Q^{2}\right)_{\gamma \rightarrow \eta^{\prime}}$ are consistent with the pQCD expectations. The photon to meson transition form factor is the simplest QCD hadronic exclusive amplitude, and thus it is critical to understand this discrepancy. As we shall discuss below, AdS/QCD predicts a broad distribution amplitude $\phi_{\pi}(x, Q)$ in the nonperturbative domain, but since ERBL evolution leads to a narrower distribution in the high $Q$ domain, it cannot explain the BaBar anomaly. It is hard to imagine that the pion distribution amplitude is very flat [28, 29, 30] since this corresponds to a pointlike non-composite hadron. It is crucial to measure $\frac{d \sigma}{d t}\left(\gamma \gamma \rightarrow \pi^{0} \pi^{0}\right)$ since the $\mathrm{CM}$ angular distribution is very sensitive to the shape of $\phi_{\pi}(x, Q)$ [31].

The Hall A collaboration [32] at JLab has reported another significant exception to the general empirical success of dimensional counting in fixed-CM-angle Compton scattering $d \sigma / d t(\gamma p \rightarrow \gamma p) \sim F\left(\theta_{C M}\right) / s^{8}$ instead of the predicted $1 / s^{6}$ scaling. The deviations from fixed-angle conformal scaling may be due to corrections from resonance contributions in the JLab energy range. It is interesting that the hadron form factor $R_{V}(t)$ [33], which multiplies the $\gamma q \rightarrow \gamma q$ amplitude is found by Hall A to scale as $1 / t^{2}$, in agreement with the pQCD and AdS/QCD prediction. In addition, the Belle measurement 34] of the timelike two-photon cross section $d \sigma / d t(\gamma \gamma \rightarrow p \bar{p})$ is consistent with $1 / s^{6}$ scaling. 
Although large-angle proton-proton elastic scattering is well described by dimensional scaling $s^{10} d \sigma / d t(p p \rightarrow p p) \sim$ constant at fixed CM angle, extraordinarily large spin-spin correlations are observed [35]. The ratio of scattering cross sections for spinparallel and normal to the scattering plane versus spin-antiparallel reaches $R_{N N} \simeq 4$ in large angle $p p \rightarrow p p$ at $\sqrt{s} \simeq 5 \mathrm{GeV}$; this is a remarkable example of "exclusive transversity". Color transparency is observed at lower energies but it fails [36] at the same energy where $R_{N N}$ becomes large. In fact, these anomalies have a natural explanation [37] as a resonance effect related to the charm threshold in $p p$ scattering. Alternative explanations of the large spin correlation are discussed and reviewed in Ref. [38]. Resonance formation is a natural phenomenon when all constituents are relatively at rest. For example, a resonance effect can occur due to the intermediate state $u u d u u d c \bar{c}$ at the charm threshold $\sqrt{s}=5 \mathrm{GeV}$ in $p p$ collisions. Since the $c$ and $\bar{c}$ have opposite intrinsic parity, the resonance appears in the $L=J=S=1$ partial wave for $p p \rightarrow p p$ which is only allowed for spin-parallel and normal to the scattering plane $A_{N N}=1$ [37]. Resonance formation at the charm threshold also explains the dramatic quenching of color transparency seen in quasielastic pn scattering by the EVA BNL experiment [36] in the same kinematic region. The reason why these effects are apparent in $p p \rightarrow p p$ scattering is that the amplitude for the formation of an $u$ uduudc $\bar{c} s$-channel resonance in the intermediate state is of the same magnitude as the fast-falling background $p p \rightarrow p p$ pQCD amplitude from quark interchange at large CM angles: $M(p p \rightarrow p p) \sim 1 / u^{2} t^{2}$. The open charm cross section in $p p$ scattering is predicted by unitarity to be of order of $1 \mu b$ at threshold [37]. One also expects similar novel QCD phenomena in large-angle photoproduction $\gamma p \rightarrow \pi N$ near the charm threshold, including the breakdown of color transparency and strong spin-spin correlations. These effects can be tested by measurements at the new JLab $12 \mathrm{GeV}$ facility, which would confirm resonance formation in a low partial wave in $\gamma p \rightarrow \pi N$ at $\sqrt{s} \simeq 4 \mathrm{GeV}$ due to attractive forces in the $u u d \bar{c} c$ channel.

Another difficulty for the application of pQCD to exclusive processes is the famous $J / \psi \rightarrow \rho \pi$ puzzle; the observed unusually large branching ratio for $J / \psi \rightarrow \rho \pi$. In contrast, the branching ratio for $\Psi^{\prime} \rightarrow \rho \pi$ is very small. Such decays into pseudoscalar plus vector mesons require light-quark helicity suppression or internal orbital angular momentum and thus should be suppressed by hadron helicity conservation in pQCD. However, the $J / \psi \rightarrow \rho \pi$ puzzle can be explained by the presence of intrinsic charm Fock states in the outgoing mesons [39]. 


\section{Light-Front Quantization and Exclusive Processes}

Light-front (LF) quantization is the ideal framework for describing the structure of hadrons in terms of their quark and gluon degrees of freedom. The light-front wavefunctions (LFWFs) of bound states in QCD are relativistic generalizations of the Schrödinger wavefunctions, but they are determined at fixed light-front time $\tau=x^{+}=x^{0}+x^{3}$, the time marked by the front of a light wave [40], rather than at fixed ordinary time $t$. They play the same role in hadron physics that Schrödinger wavefunctions play in atomic physics. In addition, the simple structure of the LF vacuum provides an unambiguous definition of the partonic content of a hadron in QCD. In the light-front formalism, one sets boundary conditions at fixed $\tau$ and then evolves the system using the LF Hamiltonian $P^{-}=P^{0}-P^{3}=i d / d \tau$. The invariant Hamiltonian $H_{L F}=P_{\mu} P^{\mu}=P^{+} P^{-}-P_{\perp}^{2}$ has eigenvalues $\mathcal{M}^{2}$ where $\mathcal{M}$ is the physical hadron mass. The Heisenberg equation for QCD on the light-front thus takes the form $H_{L F}\left|\Psi_{H}\right\rangle=\mathcal{M}_{H}^{2}\left|\Psi_{H}\right\rangle$, where $H_{L F}$ is determined canonically from the QCD Lagrangian. Its eigenfunctions are the light-front eigenstates which define the frame-independent light-front wavefunctions, and its eigenvalues yield the hadronic spectrum, the bound states as well as the continuum. The projection of the eigensolutions on the free Fock basis gives the $n$-parton LF wavefunctions $\psi_{n / H}=\left\langle n \mid \Psi_{H}\right\rangle$ needed for phenomenology. Heisenberg's problem on the light-front can be solved numerically using discretized light-front quantization (DLCQ) [41] by applying antiperiodic boundary conditions in $\sigma=x^{0}-x^{3}$. This method has been used successfully to solve many lower dimension quantum field theories [42].

The light-front Fock state wavefunctions $\psi_{n / H}\left(x_{i}, \vec{k}_{\perp}, \lambda_{i}\right)$ are functions of LF momentum fractions $x_{i}=\frac{k_{i}^{+}}{P^{+}}=\frac{k_{i}^{0}+k_{i}^{3}}{P^{0}+P^{3}}$ with $\sum_{i=1}^{n} x_{i}=1$, relative transverse momenta satisfying $\sum_{i=1}^{n} \vec{k}_{\perp i}=0$, and spin projections $\lambda_{i}$. Remarkably, the LFWFs are independent of the hadron's total momentum $P^{+}=P^{0}+P^{3}$, so that once they are known in one frame, they are known in all frames; Wigner transformations and Melosh rotations are not required. The light-front formalism for gauge theories in light-cone gauge is particularly useful in that there are no ghosts and one has a direct physical interpretation of orbital angular momentum. They also allow one to formulate hadronization in inclusive and exclusive reactions at the amplitude level.

A key example of the utility of the light-front is the Drell-Yan West formula [43, 44] for the spacelike form factors of electromagnetic currents given as overlaps of initial and final LFWFs. At high momentum where one can iterate the hard scattering kernel, this yields the dimensional counting rules, factorization theorems, and ERBL evolution of the distribution amplitudes. The gauge-invariant distribution amplitudes $\phi_{H}\left(x_{i}, Q\right)$ defined from the integral over the transverse momenta $\mathbf{k}_{\perp i}^{2} \leq Q^{2}$ of the valence (smallest $n$ ) Fock state provide a fundamental measure of the hadron at the 
amplitude level [3, 4]; they are the nonperturbative inputs to the factorized form of hard exclusive amplitudes and exclusive heavy hadron decays in pQCD.

Given the light-front wavefunctions $\psi_{n / H}$ one can compute a large range of other hadron observables. For example, the valence and sea quark and gluon distributions which are measured in deep inelastic lepton scattering are defined from the squares of the LFWFs summed over all Fock states $n$. Exclusive weak transition amplitudes [45] such as $B \rightarrow \ell \nu \pi$, and the generalized parton distributions [46] measured in deeply virtual Compton scattering $\gamma^{*} p \rightarrow \gamma p$ are (assuming the "handbag" approximation) overlaps of the initial and final LFWFs with $n=n^{\prime}$ and $n=n^{\prime}+2$. The resulting distributions obey the DGLAP and ERBL evolution equations as a function of the maximal invariant mass, thus providing a physical factorization scheme [47]. In each case, the derived quantities satisfy the appropriate operator product expansions, sum rules, and evolution equations. At large $x$ where the struck quark is far-off shell, DGLAP evolution is quenched [48], so that the fall-off of the DIS cross sections in $Q^{2}$ satisfies Bloom-Gilman inclusive-exclusive duality at fixed $W^{2}$.

The simple features of the light-front contrast with the conventional instant form where one quantizes at $t=0$. For example, calculating a hadronic form factor requires boosting the hadron's wavefunction from the initial to final state, a dynamical problem as difficult as solving QCD itself. Moreover current matrix elements require computing the interaction of the probe with all of connected currents fluctuating in the QCD vacuum. Each contributing diagram is frame-dependent.

A fundamental theorem for gravity can be derived from the equivalence principle: the anomalous gravitomagnetic moment defined from the spin-flip matrix element of the energy-momentum tensor is identically zero $B(0)=0$ [49]. This theorem can be proven in the light-front formalism Fock state by Fock state [50]. The LF vacuum is trivial up to zero modes in the front form, thus eliminating contributions to the cosmological constant from QED or QCD [51].

\section{AdS/QCD}

One of the most significant theoretical advances in recent years has been the application of the AdS/CFT correspondence [52] between string theories defined in 5dimensional Anti-de Sitter (AdS) space-time and conformal field theories in physical space-time, to study the dynamics of strongly coupled quantum field theories. The essential principle underlying the AdS/CFT approach to conformal gauge theories is the isomorphism of the group of Poincaré and conformal transformations $S O(4,2)$ to the group of isometries of Anti-de Sitter space. The AdS metric is

$$
d s^{2}=\frac{R^{2}}{z^{2}}\left(\eta_{\mu \nu} d x^{\mu} d x^{\nu}-d z^{2}\right),
$$

which is invariant under scale changes of the coordinate in the fifth dimension $z \rightarrow \lambda z$ and $x_{\mu} \rightarrow \lambda x_{\mu}$. Thus one can match scale transformations of the theory in $3+1$ 
physical space-time to scale transformations in the fifth dimension $z$. In the AdS/CFT duality, the amplitude $\Phi(z)$ represents the extension of the hadron into the additional fifth dimension. The behavior of $\Phi(z) \rightarrow z^{\tau}$ at $z \rightarrow 0$ matches the twist-dimension $\tau$ of the hadron at short distances.

QCD is not conformal but there is in fact much empirical evidence from lattice gauge theory [53], Dyson Schwinger theory [54], and empirical effective charges [55], that the QCD $\beta$-function vanishes in the infrared [56]. The QCD infrared fixed point arises since the propagators of the confined quarks and gluons in the loop integrals contributing to the $\beta$-function have a maximal wavelength [57]. The decoupling of quantum loops in the infrared is analogous to QED where vacuum polarization corrections to the photon propagator decouple at $Q^{2} \rightarrow 0$. Since there is a window where the QCD coupling is large and approximately constant, QCD resembles a conformal theory for massless quarks. Thus, even though QCD is not conformally invariant, one can use the mathematical representation of the conformal group in five-dimensional Anti-de Sitter space to construct an analytic first approximation to the theory.

The AdS/QCD correspondence is now providing important insight into the properties of QCD needed to compute exclusive reactions. In particular, the soft-wall AdS/QCD model modified by a positive sign dilaton metric, which represents color confinement, leads to a remarkable one-parameter description of nonperturbative hadron dynamics, including successful predictions for the meson and baryon spectrum for zero quark mass, including a zero-mass pion, a Regge spectrum of linear trajectories with the same slope in orbital angular $L$ and the principal quantum number $n$, as well as dynamical form factors. The theory predicts dimensional counting for form factors and other fixed CM angle exclusive reactions. Moreover, as we shall review, light-front holography allows one to map the hadronic amplitudes $\phi_{H}(z)$ determined in the AdS fifth dimension $z$ to the valence LFWFs of each hadron as a function of a covariant impact variable $\zeta$. Moreover, the same techniques provide a prediction for the QCD coupling $\alpha_{s}\left(Q^{2}\right)$ and its $\beta$-function which reflects the dynamics of confinement.

\section{AdS/QCD Models}

We thus begin with a conformal approximation to QCD to model an effective dual gravity description in AdS space. The five-dimensional $\mathrm{AdS}_{5}$ geometrical representation of the conformal group represents scale transformations within the conformal window. Confinement can be introduced with a sharp cut-off in the infrared region of AdS space, as in the "hard-wall" model [5], or, more successfully, using a dilaton background in the fifth dimension to produce a smooth cutoff at large distances as in the "soft-wall" model [58]. We assume a dilaton profile $\exp \left(+\kappa^{2} z^{2}\right)$ [59, 60, 61, 62], with opposite sign to that of Ref. [58. The soft-wall AdS/QCD model with a

positive-sign dilaton-modified AdS metric, [60] $d s^{2}=\left(R^{2} / z^{2}\right) e^{+\kappa^{2} z^{2}}\left(\eta_{\mu \nu} d x^{\mu} d x^{\nu}-d z^{2}\right)$, 
leads to the harmonic potential [59] $U(z)=\kappa^{4} z^{2}+2 \kappa^{2}(L+S-1)$ in the fifth dimension coordinate $z$. the resulting spectrum reproduces linear Regge trajectories. $\mathcal{M}^{2}(S, L, n)=4 \kappa^{2}(n+L+S / 2)$, where $\mathcal{M}^{2}(S, L, n)$ is proportional to the internal spin, orbital angular momentum $L$ and the principal radial quantum number $n$.

The modified metric induced by the dilaton can be interpreted in AdS space as a gravitational potential for an object of mass $m$ in the fifth dimension: $V(z)=$ $m c^{2} \sqrt{g_{00}}=m c^{2} R e^{ \pm \kappa^{2} z^{2} / 2} / z$. In the case of the negative solution, the potential decreases monotonically, and thus an object in AdS will fall to infinitely large values of $z$. For the positive solution, the potential is non-monotonic and has an absolute minimum at $z_{0}=1 / \kappa$. Furthermore, for large values of $z$ the gravitational potential increases exponentially, confining any object to distances $\langle z\rangle \sim 1 / \kappa[59$. We thus will choose the confining positive sign dilaton solution [59, 60] with opposite sign to that of Ref. [58]. This additional warp factor leads to a well-defined scale-dependent effective coupling.

Glazek and Schaden [63] have shown that a harmonic oscillator confining potential naturally arises as an effective potential between heavy quark states when one stochastically eliminates higher gluonic Fock states. Also, Hoyer [64] has argued that the Coulomb and linear potentials are uniquely allowed in the Dirac equation at the classical level. The linear potential becomes a harmonic oscillator potential in the corresponding Klein-Gordon equation.

\section{Light-Front Holography}

Light-front holography [65, 66, 67, 68, 69] connects the equations of motion in AdS space and the Hamiltonian formulation of QCD in physical space-time quantized on the light front at fixed LF time. This correspondence provides a direct connection between the hadronic amplitudes $\Phi(z)$ in AdS space with LF wavefunctions $\phi(\zeta)$ describing the quark and gluon constituent structure of hadrons in physical spacetime. In the case of a meson, $\zeta=\sqrt{x(1-x) \mathbf{b}_{\perp}^{2}}$ is a Lorentz invariant coordinate which measures the distance between the quark and antiquark; it is analogous to the radial coordinate $r$ in the Schrödinger equation. Here $\vec{b}_{\perp}$ is the Fourier conjugate of the transverse momentum $\vec{k}_{\perp}$. In effect $\zeta$ represents the off-light-front energy shell and invariant mass dependence of the bound state; it allows the separation of the dynamics of quark and gluon binding from the kinematics of constituent spin and internal orbital angular momentum [65]. Light-front holography thus provides a connection between the description of hadronic modes in AdS space and the Hamiltonian formulation of QCD in physical space-time quantized on the light-front at fixed LF time $\tau$. The resulting equation for the mesonic $q \bar{q}$ bound states at fixed light-front time has the form of a single-variable relativistic Lorentz invariant Schrödinger equation [65]

$$
\left(-\frac{d^{2}}{d \zeta^{2}}-\frac{1-4 L^{2}}{4 \zeta^{2}}+U(\zeta)\right) \phi(\zeta)=\mathcal{M}^{2} \phi(\zeta)
$$


where the confining potential is $U(\zeta)=\kappa^{4} \zeta^{2}+2 \kappa^{2}(L+S-1)$ in the soft-wall model with a positive-sign dilaton-modified AdS metric [59]. Its eigenvalues determine the hadronic spectra and its eigenfunctions are related to the light-front wavefunctions of hadrons for general spin and orbital angular momentum. This LF wave equation serves as a semiclassical first approximation to QCD, and it is equivalent to the equations of motion which describe the propagation of spin- $J$ modes in AdS space. The resulting light-front wavefunctions provide a fundamental description of the structure and internal dynamics of hadronic states in terms of their constituent quark and gluons. There is only one parameter, the mass scale $\kappa \sim 1 / 2 \mathrm{GeV}$, which enters the confinement potential. In the case of mesons $S=0,1$ is the combined spin of the $q$ and $\bar{q}$ state, $L$ is their relative orbital angular momentum as determined by the hadronic light-front wavefunctions.

The mapping between the LF invariant variable $\zeta$ and the fifth-dimension AdS coordinate $z$ was originally obtained by matching the expression for electromagnetic current matrix elements in AdS space with the corresponding expression for the current matrix element, using LF theory in physical space time [66]. It has also been shown that one obtains the identical holographic mapping using the matrix elements of the energy-momentum tensor [68, 70], thus verifying the consistency of the holographic mapping from AdS to physical observables defined on the light front.

\section{The Hadron Spectrum and Form Factors in Light- Front AdS/QCD}

The meson spectrum predicted by Eq. 2 has a string-theory Regge form $\mathcal{M}^{2}=$ $4 \kappa^{2}(n+L+S / 2)$; i.e., the square of the eigenmasses are linear in both the orbital angular momentum $L$ and $n$, where $n$ counts the number of nodes of the wavefunction in the radial variable $\zeta$. The spectrum also depends on the internal spin S. This is illustrated for the pseudoscalar and vector meson spectra in Fig. 1, where the data are from Ref. [71]. The pion $(S=0, n=0, L=0)$ is massless for zero quark mass, consistent with the chiral invariance of massless quarks in QCD. Thus one can compute the hadron spectrum by simply adding $4 \kappa^{2}$ for a unit change in the radial quantum number, $4 \kappa^{2}$ for a change in one unit in the orbital quantum number $L$ and $2 \kappa^{2}$ for a change of one unit of spin $S$. Remarkably, the same rule holds for three-quark baryons as we shall show below.

The eigensolutions of Eq. 2 provide the light-front wavefunctions of the valence Fock state of the hadrons $\psi\left(x, \vec{b}_{\perp}\right)$ as illustrated for the pion in Fig. 2 for the softwall (a) and hard-wall (b) models. The resulting distribution amplitude has a broad form $\phi_{\pi}(x) \sim \sqrt{x(1-x)}$ which is compatible with moments determined from lattice gauge theory. One can then immediately compute observables such as hadronic form factors (overlaps of LFWFs), structure functions (squares of LFWFs), as well as the generalized parton distributions and distribution amplitudes which underly hard 

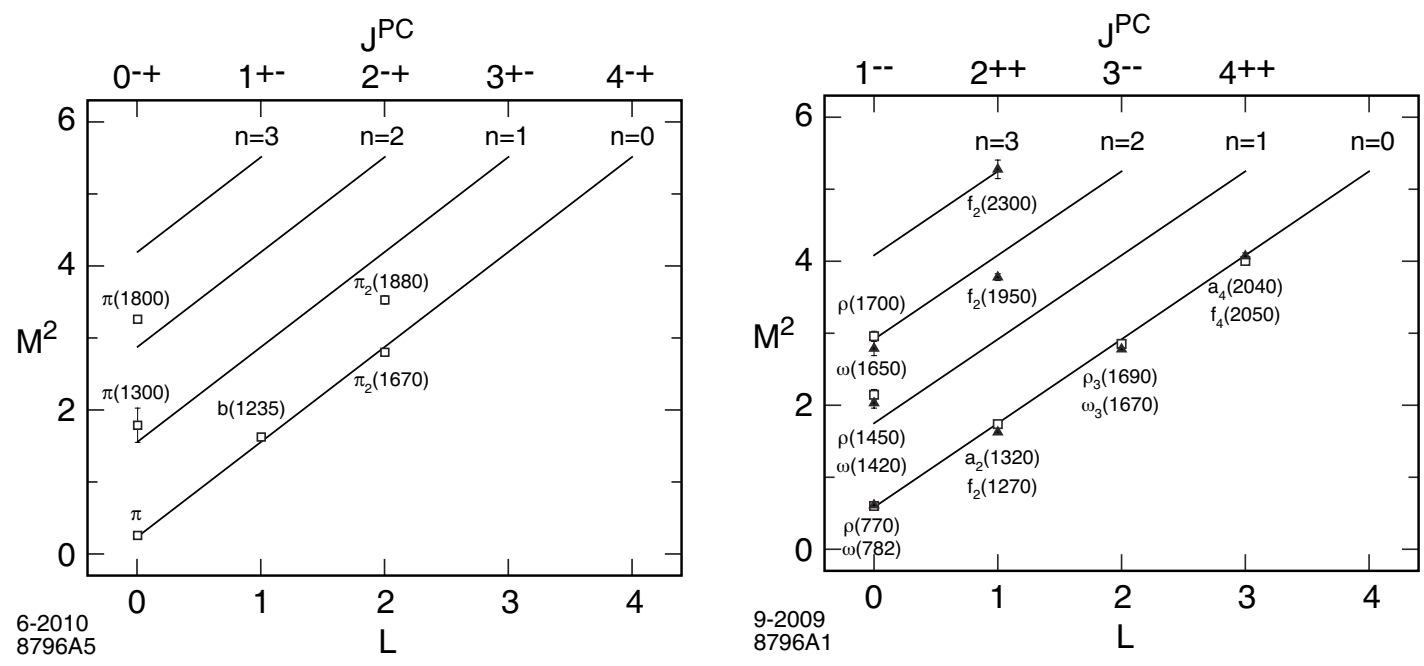

Figure 1: Parent and daughter Regge trajectories for (a) the $\pi$-meson family with $\kappa=0.6 \mathrm{GeV}$; and (b) the $I=1 \rho$-meson and $I=0 \omega$-meson families with $\kappa=0.54$ $\mathrm{GeV}$.

exclusive reactions. For example, hadronic form factors can be predicted from the overlap of LFWFs in the Drell-Yan West formula. The prediction for the space-like pion form factor is shown in Fig. 2 (c). The pion form factor and the vector meson poles residing in the dressed current in the soft wall model require choosing a value of $\kappa$ smaller by a factor of $1 / \sqrt{2}$ than the canonical value of $\kappa$ which determines the mass scale of the hadronic spectra. This shift is apparently due to the fact that the transverse current in $e^{+} e^{-} \rightarrow q \bar{q}$ creates a quark pair with $L^{z}= \pm 1$ instead of the $L^{z}=0 q \bar{q}$ composition of the vector mesons in the spectrum.

Individual hadrons in AdS/QCD are identified by matching the power behavior of the hadronic amplitude at the AdS boundary at small $z$ to the twist $\tau$ of its interpolating operator at short distances as required by the AdS/CFT dictionary. The twist also equals the dimension of fields appearing in chiral super-multiplets [75]; thus the twist of a hadron equals the number of constituents plus the relative orbital angular momentum. One then can apply light-front holography to relate the amplitude eigensolutions in the fifth-dimension coordinate $z$ to the LF wavefunctions in the physical space-time variable $\zeta$.

Equation (2) was derived by taking the LF bound-state Hamiltonian equation of motion as the starting point [65]. The term $L^{2} / \zeta^{2}$ in the LF equation of motion (2) is derived from the reduction of the LF kinetic energy when one transforms to two-dimensional cylindrical coordinates $(\zeta, \varphi)$, in analogy to the $\ell(\ell+1) / r^{2}$ Casimir term in Schrödinger theory. One thus establishes the interpretation of $L$ in the AdS equations of motion. The interaction terms build confinement corresponding to the dilaton modification of AdS space [65]. The duality between these two methods 

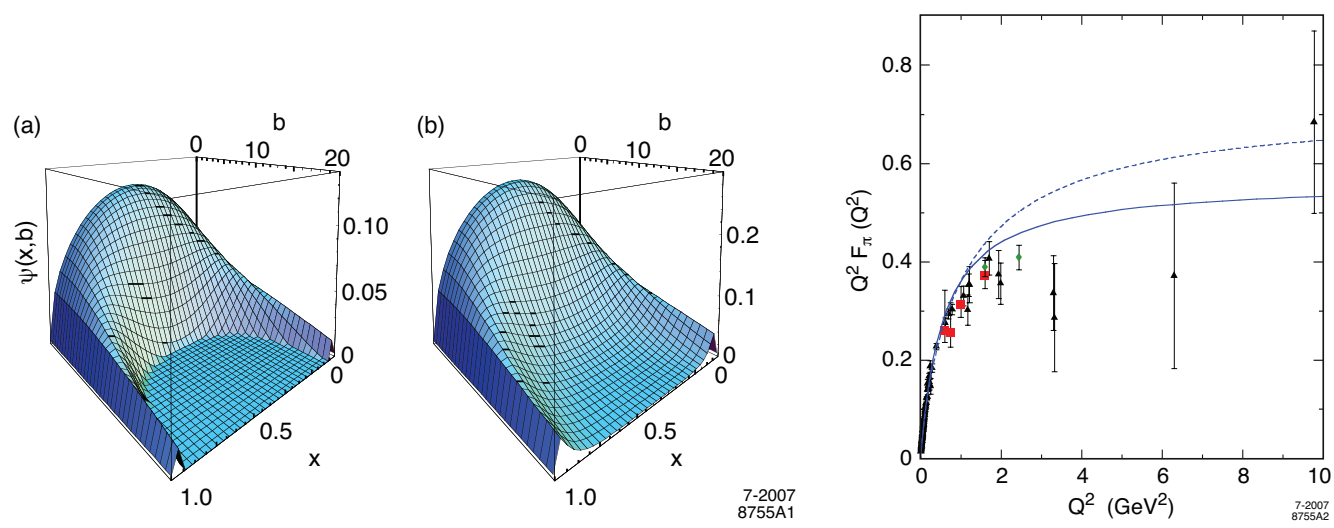

Figure 2: Pion LF wavefunction $\psi_{\pi}\left(x, \vec{b}_{\perp}\right)$ for the AdS/QCD (a) hard-wall $\left(\Lambda_{Q C D}=\right.$ $0.32 \mathrm{GeV}$ ) and (b) soft-wall $(\kappa=0.375 \mathrm{GeV})$ models. (c) Space-like scaling behavior for $Q^{2} F_{\pi}\left(Q^{2}\right)$. The continuous line is the prediction of the soft-wall model for $\kappa=$ $0.375 \mathrm{GeV}$. The dashed line is the prediction of the hard-wall model for $\Lambda_{\mathrm{QCD}}=0.22$ $\mathrm{GeV}$. The triangles are the data compilation of Baldini et al. [72], the boxes are JLAB 1 data [73] and the diamonds are JLAB 2 data [74].

provides a direct connection between the description of hadronic modes in AdS space and the Hamiltonian formulation of QCD in physical space-time quantized on the light-front at fixed LF time $\tau$.

The identification of orbital angular momentum of the constituents is a key element in the description of the internal structure of hadrons using holographic principles. In our approach quark and gluon degrees of freedom are explicitly introduced in the gauge/gravity correspondence [76], in contrast with the usual AdS/QCD framework [77, 78] where axial and vector currents become the primary entities as in effective chiral theory. Unlike the top-down string theory approach, one is not limited to hadrons of maximum spin $J \leq 2$, and one can study baryons with finite color $N_{C}=3$. Higher spin modes follow from shifting dimensions in the AdS wave equations. In the soft-wall model the usual Regge behavior is found $\mathcal{M}^{2} \sim n+L$, predicting the same multiplicity of states for mesons and baryons as observed experimentally [79]. It is possible to extend the model to hadrons with heavy quark constituents by introducing nonzero quark masses and short-range Coulomb corrections. For other recent calculations of the hadronic spectrum based on AdS/QCD, see Refs. [80, 81, 82, 83, 84, 85, 86, 87, 88, 89, 90, 91, 92, 93, 94. Other recent computations of the pion form factor are given in Refs. [95, 96, 97].

For baryons, the light-front wave equation is a linear equation determined by the LF transformation properties of spin $1 / 2$ states. A linear confining potential $U(\zeta) \sim \kappa^{2} \zeta$ in the LF Dirac equation leads to linear Regge trajectories [98]. For fermionic modes the light-front matrix Hamiltonian eigenvalue equation $D_{L F}|\psi\rangle=$ $\mathcal{M}|\psi\rangle, H_{L F}=D_{L F}^{2}$, in a $2 \times 2$ spinor component representation is equivalent to the 
system of coupled linear equations

$$
\begin{aligned}
-\frac{d}{d \zeta} \psi_{-}-\frac{\nu+\frac{1}{2}}{\zeta} \psi_{-}-\kappa^{2} \zeta \psi_{-} & =\mathcal{M} \psi_{+}, \\
\frac{d}{d \zeta} \psi_{+}-\frac{\nu+\frac{1}{2}}{\zeta} \psi_{+}-\kappa^{2} \zeta \psi_{+} & =\mathcal{M} \psi_{-},
\end{aligned}
$$

with eigenfunctions

$$
\begin{aligned}
& \psi_{+}(\zeta) \sim z^{\frac{1}{2}+\nu} e^{-\kappa^{2} \zeta^{2} / 2} L_{n}^{\nu}\left(\kappa^{2} \zeta^{2}\right) \\
& \psi_{-}(\zeta) \sim z^{\frac{3}{2}+\nu} e^{-\kappa^{2} \zeta^{2} / 2} L_{n}^{\nu+1}\left(\kappa^{2} \zeta^{2}\right)
\end{aligned}
$$

and eigenvalues $\mathcal{M}^{2}=4 \kappa^{2}(n+\nu+1)$.

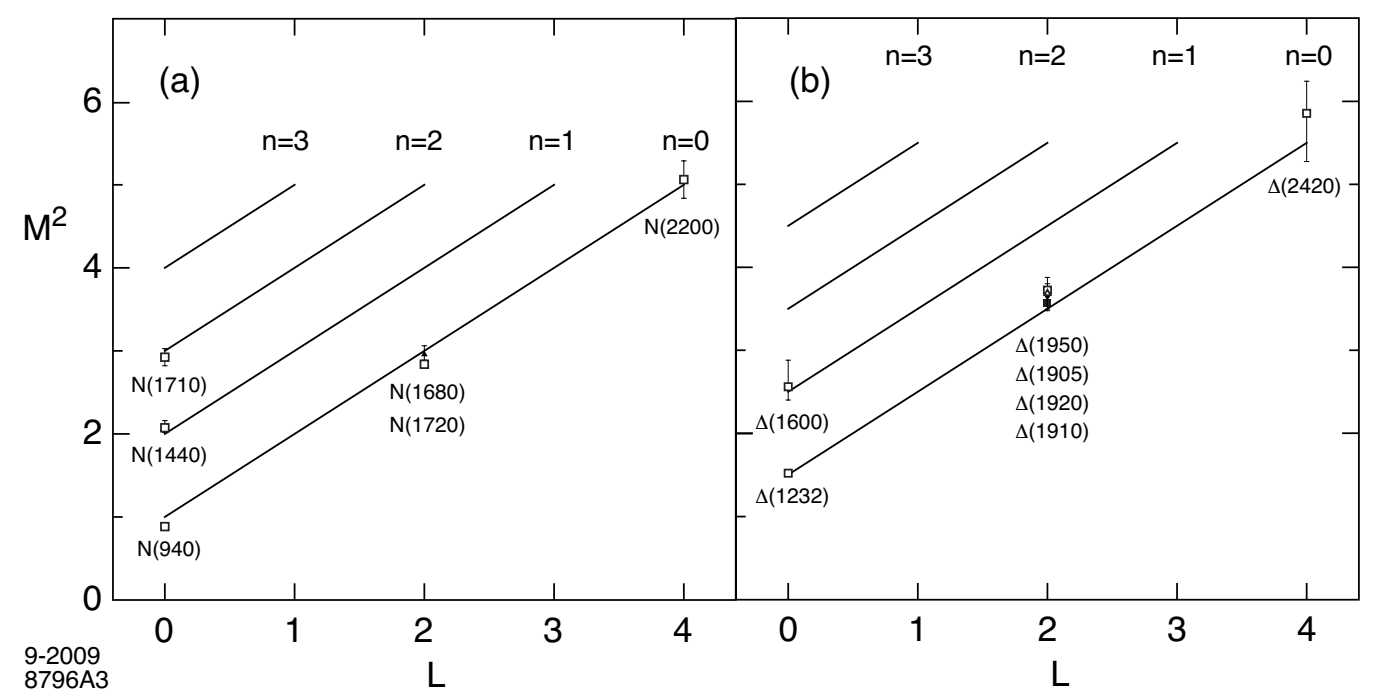

Figure 3: 56-plet Regge trajectories for the $N$ and $\Delta$ baryon families for $\kappa=0.5$ $\mathrm{GeV}$.

The baryon interpolating operator $\mathcal{O}_{3+L}=\psi D_{\left\{\ell_{1}\right.} \ldots D_{\ell_{q}} \psi D_{\ell_{q+1}} \ldots D_{\left.\ell_{m}\right\}} \psi, L=$ $\sum_{i=1}^{m} \ell_{i}$, is a twist 3 , dimension $9 / 2+L$ with scaling behavior given by its twistdimension $3+L$. We thus require $\nu=L+1$ to match the short distance scaling behavior. Higher spin modes are obtained by shifting dimensions for the fields. Thus, as in the meson sector, the increase in the mass squared for higher baryonic state is $\Delta n=4 \kappa^{2}, \Delta L=4 \kappa^{2}$ and $\Delta S=2 \kappa^{2}$, relative to the lowest ground state, the proton. Since our starting point to find the bound state equation of motion for baryons is the light-front, we fix the overall energy scale identical for mesons and baryons by imposing chiral symmetry to the pion [69] in the LF Hamiltonian equations. By contrast, if we start with a five-dimensional action for a scalar field in presence of a positive sign dilaton, the pion is automatically massless. 
The predictions for the 56-plet of light baryons under the $S U(6)$ flavor group are shown in Fig. 3. As for the predictions for mesons in Fig. 1, only confirmed PDG [71] states are shown. The Roper state $N(1440)$ and the $N(1710)$ are well accounted for in this model as the first and second radial states. Likewise the $\Delta(1660)$ corresponds to the first radial state of the $\Delta$ family. The model is successful in explaining the important parity degeneracy observed in the light baryon spectrum, such as the $L=2, N(1680)-N(1720)$ degenerate pair and the $L=2$, $\Delta(1905), \Delta(1910), \Delta(1920), \Delta(1950)$ states which are degenerate within error bars. Parity degeneracy of baryons is also a property of the hard wall model, but radial states are not well described in this model [99].

As an example of the scaling behavior of a twist $\tau=3$ hadron, we compute the spin non-flip nucleon form factor in the soft wall model [98]. The proton and neutron Dirac form factors are given by

$$
\begin{gathered}
F_{1}^{p}\left(Q^{2}\right)=\int d \zeta J(Q, \zeta)\left|\psi_{+}(\zeta)\right|^{2} \\
F_{1}^{n}\left(Q^{2}\right)=-\frac{1}{3} \int d \zeta J(Q, \zeta)\left[\left|\psi_{+}(\zeta)\right|^{2}-\left|\psi_{-}(\zeta)\right|^{2}\right],
\end{gathered}
$$

where $F_{1}^{p}(0)=1, \quad F_{1}^{n}(0)=0$. The non-normalizable mode $J(Q, z)$ is the solution of the AdS wave equation for the external electromagnetic current in presence of a dilaton background $\exp \left( \pm \kappa^{2} z^{2}\right)$ [67, 100]. Plus and minus components of the twist 3 nucleon LFWF are

$$
\psi_{+}(\zeta)=\sqrt{2} \kappa^{2} \zeta^{3 / 2} e^{-\kappa^{2} \zeta^{2} / 2}, \quad \Psi_{-}(\zeta)=\kappa^{3} \zeta^{5 / 2} e^{-\kappa^{2} \zeta^{2} / 2}
$$

The results for $Q^{4} F_{1}^{p}\left(Q^{2}\right)$ and $Q^{4} F_{1}^{n}\left(Q^{2}\right)$ and are shown in Fig. 4

\section{Nonperturbative Running Coupling from Light- Front Holography}

The concept of a running coupling $\alpha_{s}\left(Q^{2}\right)$ in QCD is usually restricted to the perturbative domain. However, as in QED, it is useful to define the coupling as an analytic function valid over the full space-like and time-like domains. The study of the nonAbelian QCD coupling at small momentum transfer is a complex problem because of gluonic self-coupling and color confinement.

The definition of the running coupling in perturbative quantum field theory is scheme-dependent. As discussed by Grunberg [102], an effective coupling or charge can be defined directly from physical observables. Effective charges defined from different observables can be related to each other in the leading-twist domain using commensurate scale relations (CSR) [103]. The potential between infinitely heavy quarks can be defined analytically in momentum transfer space as the product of the 

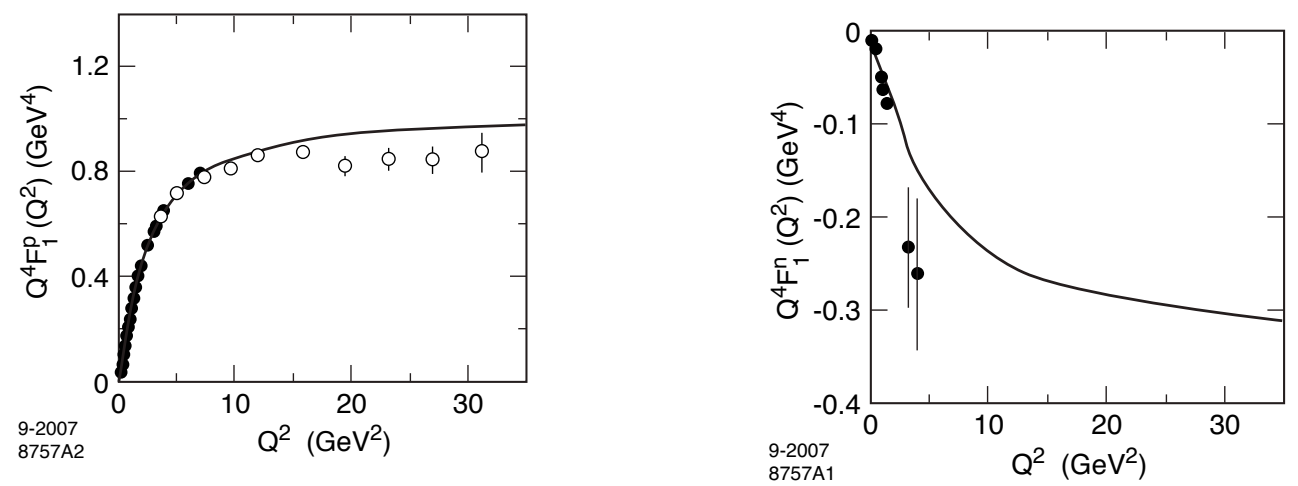

Figure 4: Predictions for $Q^{4} F_{1}^{p}\left(Q^{2}\right)$ and $Q^{4} F_{1}^{n}\left(Q^{2}\right)$ in the soft wall model for $\kappa=0.424$ $\mathrm{GeV}$ [101].

running coupling times the Born gluon propagator: $V(q)=-4 \pi C_{F} \alpha_{V}(q) / q^{2}$. This effective charge defines a renormalization scheme - the $\alpha_{V}$ scheme of Appelquist, Dine, and Muzinich [104]. In fact, the holographic coupling $\alpha_{s}^{A d S}\left(Q^{2}\right)$ can be considered to be the nonperturbative extension of the $\alpha_{V}$ effective charge defined in Ref. [104]. We can also make use of the $g_{1}$ scheme, where the strong coupling $\alpha_{g_{1}}\left(Q^{2}\right)$ is determined from the Bjorken sum rule [105]. The coupling $\alpha_{g_{1}}\left(Q^{2}\right)$ has the advantage that it is the best-measured effective charge, and it can be used to extrapolate the definition of the effective coupling to large distances [106]. Since $\alpha_{g_{1}}$ has been measured at intermediate energies, it is particularly useful for studying the transition from small to large distances.

We have recently shown [107] how the LF holographic mapping of effective classical gravity in AdS space, modified by a positive-sign dilaton background, can be used to identify an analytically simple color-confining non-perturbative effective coupling $\alpha_{s}^{A d S}\left(Q^{2}\right)$ as a function of the space-like momentum transfer $Q^{2}=-q^{2}$. This coupling incorporates confinement and agrees well with effective charge observables and lattice simulations. It also exhibits an infrared fixed point at small $Q^{2}$ and asymptotic freedom at large $Q^{2}$. However, the fall-off of $\alpha_{s}^{A d S}\left(Q^{2}\right)$ at large $Q^{2}$ is exponential: $\alpha_{s}^{A d S}\left(Q^{2}\right) \sim e^{-Q^{2} / \kappa^{2}}$, rather than the pQCD logarithmic fall-off. It agrees with hadron physics data extracted phenomenologically from different observables, as well as with the predictions of models with built-in confinement and lattice simulations. We also show that a phenomenological extended coupling can be defined which implements the pQCD behavior. The $\beta$-function derived from light-front holography becomes significantly negative in the non-perturbative regime $Q^{2} \sim \kappa^{2}$, where it reaches a minimum, signaling the transition region from the infrared (IR) conformal region, characterized by hadronic degrees of freedom, to a pQCD conformal ultraviolet (UV) regime where 
the relevant degrees of freedom are the quark and gluon constituents. The $\beta$-function vanishes at large $Q^{2}$ consistent with asymptotic freedom, and it vanishes at small $Q^{2}$ consistent with an infrared fixed point [57, 108.

Let us consider a five-dimensional gauge field $F$ propagating in $\mathrm{AdS}_{5}$ space in presence of a dilaton background $\varphi(z)$ which introduces the energy scale $\kappa$ in the five-dimensional action. At quadratic order in the field strength the action is

$$
S=-\frac{1}{4} \int d^{5} x \sqrt{g} e^{\varphi(z)} \frac{1}{g_{5}^{2}} F^{2}
$$

where the metric determinant of $\operatorname{AdS}_{5}$ is $\sqrt{g}=(R / z)^{5}, \varphi=\kappa^{2} z^{2}$ and the square of the coupling $g_{5}$ has dimensions of length. We can identify the prefactor

$$
g_{5}^{-2}(z)=e^{\varphi(z)} g_{5}^{-2}
$$

in the AdS action (8) as the effective coupling of the theory at the length scale $z$. The coupling $g_{5}(z)$ then incorporates the non-conformal dynamics of confinement. The five-dimensional coupling $g_{5}(z)$ is mapped, modulo a constant, into the Yang-Mills (YM) coupling $g_{Y M}$ of the confining theory in physical space-time using light-front holography. One identifies $z$ with the invariant impact separation variable $\zeta$ which appears in the LF Hamiltonian: $g_{5}(z) \rightarrow g_{Y M}(\zeta)$. Thus

$$
\alpha_{s}^{A d S}(\zeta)=g_{Y M}^{2}(\zeta) / 4 \pi \propto e^{-\kappa^{2} \zeta^{2}}
$$

In contrast with the 3-dimensional radial coordinates of the non-relativistic Schrödinger theory, the natural light-front variables are the two-dimensional cylindrical coordinates $(\zeta, \phi)$ and the light-cone fraction $x$. The physical coupling measured at the scale $Q$ is the two-dimensional Fourier transform of the LF transverse coupling $\alpha_{s}^{A d S}(\zeta)(10$. Integration over the azimuthal angle $\phi$ gives the Bessel transform

$$
\alpha_{s}^{A d S}\left(Q^{2}\right) \sim \int_{0}^{\infty} \zeta d \zeta J_{0}(\zeta Q) \alpha_{s}^{A d S}(\zeta)
$$

in the $q^{+}=0$ light-front frame where $Q^{2}=-q^{2}=-\mathbf{q}_{\perp}^{2}>0$ is the square of the spacelike four-momentum transferred to the hadronic bound state. Using this ansatz we then have from Eq. (11)

$$
\alpha_{s}^{A d S}\left(Q^{2}\right)=\alpha_{s}^{A d S}(0) e^{-Q^{2} / 4 \kappa^{2}}
$$

In contrast, the negative dilaton solution $\varphi=-\kappa^{2} z^{2}$ leads to an integral which diverges at large $\zeta$. We identify $\alpha_{s}^{A d S}\left(Q^{2}\right)$ with the physical QCD running coupling in its nonperturbative domain.

The flow equation (9) from the scale dependent measure for the gauge fields can be understood as a consequence of field-strength renormalization. In physical QCD we can rescale the non-Abelian gluon field $A^{\mu} \rightarrow \lambda A^{\mu}$ and field strength $G^{\mu \nu} \rightarrow \lambda G^{\mu \nu}$ 
in the QCD Lagrangian density $\mathcal{L}_{\mathrm{QCD}}$ by a compensating rescaling of the coupling strength $g \rightarrow \lambda^{-1} g$. The renormalization of the coupling $g_{\text {phys }}=Z_{3}^{1 / 2} g_{0}$, where $g_{0}$ is the bare coupling in the Lagrangian in the UV-regulated theory, is thus equivalent to the renormalization of the vector potential and field strength: $A_{r e n}^{\mu}=Z_{3}^{-1 / 2} A_{0}^{\mu}, G_{r e n}^{\mu \nu}=$ $Z_{3}^{-1 / 2} G_{0}^{\mu \nu}$ with a rescaled Lagrangian density $\mathcal{L}_{\mathrm{QCD}}^{r e n}=Z_{3}^{-1} \mathcal{L}_{\mathrm{QCD}}^{0}=\left(g_{\text {phys }} / g_{0}\right)^{-2} \mathcal{L}_{0}$. In lattice gauge theory, the lattice spacing $a$ serves as the UV regulator, and the renormalized QCD coupling is determined from the normalization of the gluon field strength as it appears in the gluon propagator. The inverse of the lattice size $L$ sets the mass scale of the resulting running coupling. As is the case in lattice gauge theory, color confinement in AdS/QCD reflects nonperturbative dynamics at large distances. The QCD couplings defined from lattice gauge theory and the soft wall holographic model are thus similar in concept, and both schemes are expected to have similar properties in the nonperturbative domain, up to a rescaling of their respective momentum scales.

\subsection{Comparison of the Holographic Coupling with Other Ef- fective Charges}

The effective coupling $\alpha^{A d S}\left(Q^{2}\right)$ (solid line) is compared in Fig. 5 with experimental and lattice data. For this comparison to be meaningful, we have to impose the same normalization on the AdS coupling as the $g_{1}$ coupling. This defines $\alpha_{s}^{\text {AdS }}$ normalized to the $g_{1}$ scheme: $\alpha_{g_{1}}^{A d S}\left(Q^{2}=0\right)=\pi$. Details on the comparison with other effective charges are given in Ref. [55].

The couplings in Fig. 5 (a) agree well in the strong coupling regime up to $Q \sim 1$ $\mathrm{GeV}$. The value $\kappa=0.54 \mathrm{GeV}$ is determined from the vector meson Regge trajectory [59. The lattice results shown in Fig. 5 from Ref. [53] have been scaled to match the perturbative UV domain. The effective charge $\alpha_{g_{1}}$ has been determined in Ref. [55] from several experiments. Fig. 5 also displays other couplings from different observables as well as $\alpha_{g_{1}}$ which is computed from the Bjorken sum rule [105] over a large range of momentum transfer (cyan band). At $Q^{2}=0$ one has the constraint on the slope of $\alpha_{g_{1}}$ from the Gerasimov-Drell-Hearn (GDH) sum rule [109] which is also shown in the figure. The results show no sign of a phase transition, cusp, or other non-analytical behavior, a fact which allows us to extend the functional dependence of the coupling to large distances. As discussed below, the smooth behavior of the AdS strong coupling also allows us to extrapolate its form to the perturbative domain [107].

The hadronic model obtained from the dilaton-modified AdS space provides a semi-classical first approximation to QCD. Color confinement is introduced by the harmonic oscillator potential, but effects from gluon creation and absorption are not included in this effective theory. The nonperturbative confining effects vanish exponentially at large momentum transfer (Eq. (12)), and thus the logarithmic fall-off from $\mathrm{pQCD}$ quantum loops will dominate in this regime. The running coupling $\alpha_{s}^{\text {AdS }}$ 

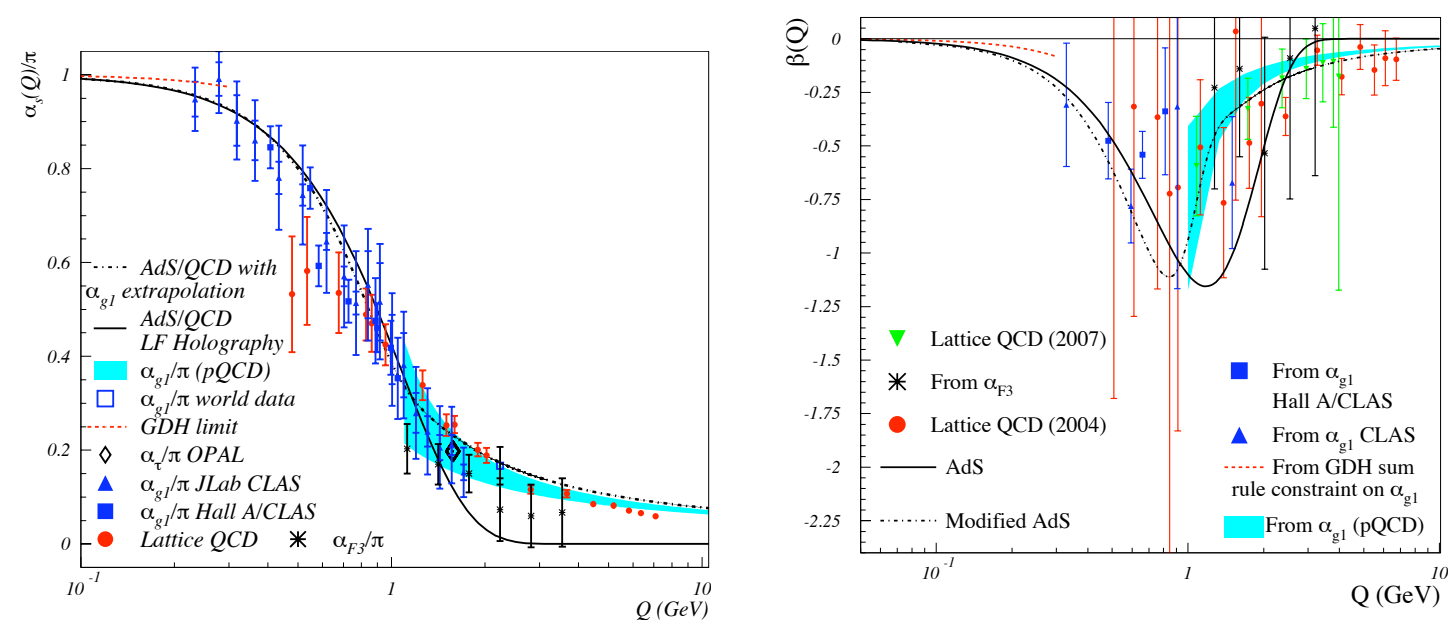

Figure 5: (a) Effective coupling from LF holography for $\kappa=0.54 \mathrm{GeV}$ compared with effective QCD couplings extracted from different observables and lattice results. (b) Prediction for the $\beta$-function compared to lattice simulations, JLab and CCFR results for the Bjorken sum rule effective charge.

given by Eq. (12) is obtained from a color-confining potential. Since the strong coupling is an analytical function of the momentum transfer at all scales, we can extend the range of applicability of $\alpha_{s}^{A d S}$ by matching to a perturbative coupling at the transition scale $Q \sim 1 \mathrm{GeV}$, where pQCD contributions become important, as described in Ref. [107]. The smoothly extrapolated result (dot-dashed line) for $\alpha_{s}$ is also shown on Fig. 5. In order to have a fully analytical model, we write

$$
\alpha_{\text {Modified, } g_{1}}^{\text {AdS }}\left(Q^{2}\right)=\alpha_{g_{1}}^{A d S}\left(Q^{2}\right) g_{+}\left(Q^{2}\right)+\alpha_{g_{1}}^{f i t}\left(Q^{2}\right) g_{-}\left(Q^{2}\right)
$$

where $g_{ \pm}\left(Q^{2}\right)=1 /\left(1+e^{ \pm\left(Q^{2}-Q_{0}^{2}\right) / \tau^{2}}\right)$ are smeared step functions which match the two regimes. The parameter $\tau$ represents the width of the transition region. Here $\alpha_{g_{1}}^{A d S}$ is given by Eq. $\sqrt{12}$ with the normalization $\alpha_{g_{1}}^{A d S}(0)=\pi$ - the plain black line in Fig. 5 - and $\alpha_{g_{1}}^{f i t}$ in Eq. (13) is the analytical fit to the measured coupling $\alpha_{g_{1}}$ [55]. The couplings are chosen to have the same normalization at $Q^{2}=0$. The smoothly extrapolated result (dot-dashed line) for $\alpha_{s}$ is also shown on Fig. 5. We use the parameters $Q_{0}^{2}=0.8 \mathrm{GeV}^{2}$ and $\tau^{2}=0.3 \mathrm{GeV}^{2}$.

\subsection{The $\beta$-Function from AdS/QCD}

The $\beta$-function for the nonperturbative effective coupling obtained from the LF holographic mapping in a positive dilaton modified AdS background is

$$
\beta^{A d S}\left(Q^{2}\right)=\frac{d}{d \log Q^{2}} \alpha^{A d S}\left(Q^{2}\right)=-\frac{\pi Q^{2}}{4 \kappa^{2}} e^{-Q^{2} /\left(4 \kappa^{2}\right)} .
$$


The solid line in Fig. 5 (b) corresponds to the light-front holographic result Eq. (14). Near $Q_{0} \simeq 2 \kappa \simeq 1 \mathrm{GeV}$, we can interpret the results as a transition from the nonperturbative IR domain to the quark and gluon degrees of freedom in the perturbative UV regime. The transition momentum scale $Q_{0}$ is compatible with the momentum transfer for the onset of scaling behavior in exclusive reactions where quark counting rules are observed [1]. For example, in deuteron photo-disintegration the onset of scaling corresponds to momentum transfer of $1.0 \mathrm{GeV}$ to the nucleon involved [110]. Dimensional counting is built into the AdS/QCD soft and hard wall models since the AdS amplitudes $\Phi(z)$ are governed by their twist scaling behavior $z^{\tau}$ at short distances, $z \rightarrow 0$ [5].

Also shown on Fig. 5 (b) are the $\beta$-functions obtained from phenomenology and lattice calculations. For clarity, we present only the LF holographic predictions, the lattice results from [53], and the experimental data supplemented by the relevant sum rules. The dot-dashed curve corresponds to the extrapolated approximation obtained by matching to AdS results to the perturbative coupling [107] given by Eq. (13). The $\beta$-function extracted from LF holography, as well as the forms obtained from the works of Cornwall [108], Bloch, Fisher et al. [111], Burkert and Ioffe [112] and Furui and Nakajima [53], are seen to have a similar shape and magnitude.

Judging from these results, we infer that the actual $\beta$-function of QCD will extrapolate between the non-perturbative results for $Q<1 \mathrm{GeV}$ and the pQCD results for $Q>1 \mathrm{GeV}$. We also observe that the general conditions

$$
\begin{gathered}
\beta(Q \rightarrow 0)=\beta(Q \rightarrow \infty)=0, \\
\beta(Q)<0, \text { for } Q>0, \\
\left.\frac{d \beta}{d Q}\right|_{Q=Q_{0}}=0, \\
\frac{d \beta}{d Q}<0, \text { for } Q<Q_{0}, \quad \frac{d \beta}{d Q}>0, \text { for } Q>Q_{0} .
\end{gathered}
$$

are satisfied by our model $\beta$-function obtained from LF holography.

Eq. (15) expresses the fact that QCD approaches a conformal theory in both the far ultraviolet and deep infrared regions. In the semiclassical approximation to QCD without particle creation or absorption, the $\beta$-function is zero and the approximate theory is scale invariant in the limit of massless quarks [113]. When quantum corrections are included, the conformal behavior is preserved at very large $Q$ because of asymptotic freedom and near $Q \rightarrow 0$ because the theory develops a fixed point. An infrared fixed point is in fact a natural consequence of color confinement [108]: since the propagators of the colored fields have a maximum wavelength, all loop integrals in the computation of the gluon self-energy decouple at $Q^{2} \rightarrow 0$ [57]. Condition (16) for $Q^{2}$ large, expresses the basic anti-screening behavior of $\mathrm{QCD}$ where the strong coupling vanishes. The $\beta$-function in $\mathrm{QCD}$ is essentially negative, thus the coupling increases monotonically from the UV to the IR where it reaches its maximum value: it has a finite value for a theory with a mass gap. Equation (17) defines the transition region at $Q_{0}$ where the $\beta$-function has a minimum. Since there is only one hadronic-partonic 
transition, the minimum is an absolute minimum; thus the additional conditions expressed in Eq (18) follow immediately from Eqs. (15, 17). The conditions given by Eqs. 15. 18 describe the essential behavior of the full $\beta$-function for an effective QCD coupling whose scheme/definition is similar to that of the $V$-scheme.

\section{Conclusions}

The combination of Anti-de Sitter space methods with light-front holography provides an accurate first approximation for the spectra and wavefunctions of meson and baryon light-quark bound states. One also obtains an elegant connection between a semiclassical first approximation to QCD, quantized on the light-front, with hadronic modes propagating on a fixed AdS background. The resulting bound-state Hamiltonian equation of motion in QCD leads to relativistic light-front wave equations in the invariant impact variable $\zeta$ which measures the separation of the quark and gluonic constituents within the hadron at equal light-front time. This corresponds to the effective single-variable relativistic Schrödinger-like equation in the AdS fifth dimension coordinate $z$, Eq. (2). The eigenvalues give the hadronic spectrum, and the eigenmodes represent the probability distributions of the hadronic constituents at a given scale. In particular, the light-front holographic mapping of effective classical gravity in AdS space, modified by a positive-sign dilaton background, provides a very good description of the spectrum and form factors of light mesons and baryons. We have also shown that the light-front holographic mapping of effective classical gravity in AdS space, modified by the positive-sign dilaton background predicts the form of a non-perturbative effective coupling $\alpha_{s}^{A d S}(Q)$ and its $\beta$-function. The AdS/QCD running coupling is in very good agreement with the effective coupling $\alpha_{g_{1}}$ extracted from the Bjorken sum rule. The holographic $\beta$-function displays a transition from nonperturbative to perturbative regimes at a momentum scale $Q \sim 1 \mathrm{GeV}$. Our analysis indicates that light-front holography captures the characteristics of the full $\beta$-function of QCD and the essential dynamics of confinement, thus giving further

support to the application of the gauge/gravity duality to the confining dynamics of strongly coupled QCD.

There are many phenomenological applications where detailed knowledge of the QCD coupling and the renormalized gluon propagator at relatively soft momentum transfer are essential. This includes exclusive and semi-exclusive processes as well as the rescattering interactions which create the leading-twist Sivers single-spin correlations in semi-inclusive deep inelastic scattering [114, 115], the Boer-Mulders functions which lead to anomalous $\cos 2 \phi$ contributions to the lepton pair angular distribution in the unpolarized Drell-Yan reaction [116], and the Sommerfeld-Sakharov-Schwinger correction to heavy quark production at threshold [117]. The confining AdS/QCD coupling from light-front holography thus can provide a quantitative understanding of this factorization-breaking physics [118]. 


\section{Acknowledgments}

We thank Volker Burkert, John Cornwall, Sadataka Furui, Philipp Hägler, Wolfgang Korsch, G. Peter Lepage, Takemichi Okui, Joannis Papavassiliou, Anatoly Radyushkin, Craig Roberts, Robert Shrock, and Peter Tandy for helpful comments. This research was supported by the Department of Energy contracts DE-AC0276SF00515 and DE-AC05-84ER40150. Invited talk, presented by SJB at the Workshop on Exclusive Reactions at High Momentum Transfer (IV), May 18-21, 2010, Thomas Jefferson National Accelerator Facility, Newport News, VA.

\section{References}

[1] S. J. Brodsky and G. R. Farrar, Phys. Rev. Lett. 31, 1153 (1973); Phys. Rev. D 11, 1309 (1975).

[2] V. A. Matveev, R. M. Muradian and A. N. Tavkhelidze, Lett. Nuovo Cim. 7, 719 (1973).

[3] G. P. Lepage and S. J. Brodsky, Phys. Lett. B 87, 359 (1979).

[4] A. V. Efremov and A. V. Radyushkin, Phys. Lett. B 94, 245 (1980).

[5] J. Polchinski and M. J. Strassler, Phys. Rev. Lett. 88, 031601 (2002) arXiv:hepth/0109174.

[6] S. J. Brodsky and A. H. Mueller, Phys. Lett. B 206, 685 (1988).

[7] G. Bertsch, S. J. Brodsky, A. S. Goldhaber and J. F. Gunion, Phys. Rev. Lett. 47, 297 (1981).

[8] J. F. Gunion, S. J. Brodsky, R. Blankenbecler, Phys. Rev. D8, 287 (1973).

[9] D. W. Sivers, S. J. Brodsky and R. Blankenbecler, Phys. Rept. 23, 1 (1976).

[10] C. G. White et al., Phys. Rev. D 49, 58 (1994).

[11] R. J. Holt, Phys. Rev. C 41, 2400 (1990).

[12] C. Bochna et al. [E89-012 Collaboration], Phys. Rev. Lett. 81, 4576 (1998) arXiv:nucl-ex/9808001.

[13] P. Rossi et al. [CLAS Collaboration], Phys. Rev. Lett. 94, 012301 (2005) arXiv:hep-ph/0405207.

[14] S. Rock et al., Phys. Rev. D 46, 24 (1992).

[15] S. J. Brodsky and B. T. Chertok, Phys. Rev. D 14, 3003 (1976). 
[16] S. J. Brodsky, C. R. Ji and G. P. Lepage, Phys. Rev. Lett. 51, 83 (1983).

[17] S. J. Brodsky and G. P. Lepage, Adv. Ser. Direct. High Energy Phys. 5, 93 (1989).

[18] E. M. Aitala et al. [E791 Collaboration], Phys. Rev. Lett. 86, 4773 (2001) arXiv:hep-ex/0010044].

[19] B. Clasie et al., arXiv:0707.1481 [nucl-ex].

[20] A. Airapetian et al. [HERMES Collaboration], Phys. Rev. Lett. 90, 052501 (2003) arXiv:hep-ex/0209072.

[21] E. L. Berger, S. J. Brodsky, Phys. Rev. Lett. 42, 940-944 (1979).

[22] E. L. Berger, T. Gottschalk, D. W. Sivers, Phys. Rev. D23, 99 (1981).

[23] E. L. Berger, S. J. Brodsky, Phys. Rev. D24, 2428 (1981).

[24] F. Arleo, S. J. Brodsky, D. S. Hwang et al., [arXiv:1006.4045 [hep-ph]].

[25] F. Arleo, S. J. Brodsky, D. S. Hwang et al., arXiv:0911.4604 [hep-ph]].

[26] S. J. Brodsky, A. Sickles, Phys. Lett. B668, 111-115 (2008) arXiv:0804.4608 [hep-ph]].

[27] V. P. Druzhinin, PoS EPS-HEP2009, 051 (2009) arXiv:0909.3148 [hep-ex]] and preliminary results presented at IHCEP2010.

[28] A. V. Radyushkin, Phys. Rev. D80, 094009 (2009) [arXiv:0906.0323 [hep-ph]].

[29] M. V. Polyakov, JETP Lett. 90, 228-231 (2009) |arXiv:0906.0538 [hep-ph]].

[30] S. V. Mikhailov, N. G. Stefanis, Mod. Phys. Lett. A24, 2858-2867 (2009) arXiv:0910.3498 [hep-ph]].

[31] S. J. Brodsky, G. P. Lepage, Phys. Rev. D24, 1808 (1981).

[32] A. Danagoulian et al. [Hall A Collaboration], Phys. Rev. Lett. 98, 152001 (2007) arXiv:nucl-ex/0701068.

[33] M. Diehl, T. Feldmann, R. Jakob and P. Kroll, Eur. Phys. J. C 8, 409 (1999) arXiv:hep-ph/9811253|.

[34] A. Chen, International Conference on the Structure and Interactions of the Photon and 14th International Workshop on Photon-Photon Collisions (Photon 2001), Ascona, Switzerland, 2-7 Sep 2001.

[35] G. R. Court et al., Phys. Rev. Lett. 57, 507 (1986). 
[36] I. Mardor et al., Phys. Rev. Lett. 81, 5085 (1998).

[37] S. J. Brodsky and G. F. de Teramond, Phys. Rev. Lett. 60, 1924 (1988).

[38] D. Dutta and H. Gao, Phys. Rev. C 71, 032201 (2005) arXiv:hep-ph/0411267.

[39] S. J. Brodsky and M. Karliner, Phys. Rev. Lett. 78, 4682 (1997) arXiv:hepph/9704379].

[40] P. A. M. Dirac, Rev. Mod. Phys. 21, 392 (1949).

[41] H. C. Pauli and S. J. Brodsky, Phys. Rev. D 32, 2001 (1985).

[42] S. J. Brodsky, H. C. Pauli and S. S. Pinsky, Phys. Rept. 301, 299 (1998) arXiv:hep-ph/9705477.

[43] S. D. Drell and T. M. Yan, Phys. Rev. Lett. 24, 181 (1970).

[44] G. B. West, Phys. Rev. Lett. 24, 1206 (1970).

[45] S. J. Brodsky and D. S. Hwang, Nucl. Phys. B 543, 239 (1999) arXiv:hepph/9806358.

[46] S. J. Brodsky, M. Diehl and D. S. Hwang, Nucl. Phys. B 596, 99 (2001) arXiv:hep-ph/0009254.

[47] G. P. Lepage and S. J. Brodsky, Phys. Rev. D 22, 2157 (1980).

[48] S. J. Brodsky and G. P. Lepage, SLAC-PUB-2294; Workshop on Current Topics in High Energy Physics, Cal Tech., Pasadena, Calif., Feb 13-17, 1979.

[49] O. V. Teryaev, arXiv:hep-ph/9904376.

[50] S. J. Brodsky, D. S. Hwang, B. Q. Ma and I. Schmidt, Nucl. Phys. B 593, 311 (2001) arXiv:hep-th/0003082.

[51] S. J. Brodsky and R. Shrock, arXiv:0905.1151 [hep-th]. S. J. Brodsky, C. D. Roberts, R. Shrock, and P. Tandy [arXiv:1005.4610 [nucl-th]].

[52] J. M. Maldacena, Adv. Theor. Math. Phys. 2, 231 (1998) [Int. J. Theor. Phys. 38, 1113 (1999)] arXiv:hep-th/9711200.

[53] S. Furui and H. Nakajima, Phys. Rev. D 76, 054509 (2007) arXiv:heplat/0612009].

[54] L. von Smekal, R. Alkofer and A. Hauck, Phys. Rev. Lett. 79, 3591 (1997) arXiv:hep-ph/9705242. 
[55] A. Deur, V. Burkert, J. P. Chen and W. Korsch, Phys. Lett. B 650, 244 (2007) arXiv:hep-ph/0509113.

[56] A. Deur, V. Burkert, J. P. Chen and W. Korsch, Phys. Lett. B 665, 349 (2008) arXiv:0803.4119 [hep-ph]].

[57] S. J. Brodsky and R. Shrock, Phys. Lett. B 666, 95 (2008) arXiv:0806.1535 [hep-th]].

[58] A. Karch, E. Katz, D. T. Son and M. A. Stephanov, Phys. Rev. D 74, 015005 (2006) arXiv:hep-ph/0602229].

[59] G. F. de Teramond and S. J. Brodsky, Nucl. Phys. Proc. Suppl. 199, 89 (2010) arXiv:0909.3900 [hep-ph]].

[60] O. Andreev and V. I. Zakharov, Phys. Rev. D 74, 025023 (2006) arXiv:hepph/0604204].

[61] F. Zuo, arXiv:0909.4240 [hep-ph].

[62] S. S. Afonin, arXiv:1001.3105 [hep-ph].

[63] S. D. Glazek and M. Schaden, Phys. Lett. B 198, 42 (1987).

[64] P. Hoyer, arXiv:0909.3045 [hep-ph].

[65] G. F. de Teramond and S. J. Brodsky, Phys. Rev. Lett. 102, 081601 (2009) arXiv:0809.4899 [hep-ph]].

[66] S. J. Brodsky and G. F. de Teramond, Phys. Rev. Lett. 96, 201601 (2006) arXiv:hep-ph/0602252.

[67] S. J. Brodsky and G. F. de Teramond, Phys. Rev. D 77, 056007 (2008) arXiv:0707.3859 [hep-ph]].

[68] S. J. Brodsky and G. F. de Teramond, Phys. Rev. D 78, 025032 (2008) arXiv:0804.0452 [hep-ph]].

[69] G. F. de Teramond and S. J. Brodsky, arXiv:1001.5193 [hep-ph].

[70] Z. Abidin and C. E. Carlson, Phys. Rev. D 77, 095007 (2008) arXiv:0801.3839 [hep-ph]].

[71] C. Amsler et al. (Particle Data Group), Phys. Lett. B 667, 1, (2008).

[72] R. Baldini, S. Dubnicka, P. Gauzzi, S. Pacetti, E. Pasqualucci and Y. Srivastava, Eur. Phys. J. C 11, 709 (1999). 
[73] V. Tadevosyan et al. [Jefferson Lab F(pi) Collaboration], Phys. Rev. C 75, 055205 (2007) arXiv:nucl-ex/0607007.

[74] T. Horn et al. [Fpi2 Collaboration], Phys. Rev. Lett. 97, 192001 (2006) arXiv:nucl-ex/0607005.

[75] N. J. Craig and D. Green, JHEP 0909, 113 (2009) [arXiv:0905.4088 [hep-ph]].

[76] S. J. Brodsky and G. F. de Teramond, Phys. Lett. B 582, 211 (2004) arXiv:hepth/0310227].

[77] J. Erlich, E. Katz, D. T. Son and M. A. Stephanov, Phys. Rev. Lett. 95, 261602 (2005) arXiv:hep-ph/0501128.

[78] L. Da Rold and A. Pomarol, Nucl. Phys. B 721, 79 (2005) arXiv:hepph/0501218]; JHEP 0601, 157 (2006) [arXiv:hep-ph/0510268].

[79] E. Klempt and A. Zaitsev, Phys. Rept. 454, 1 (2007) arXiv:0708.4016 [hep-ph]].

[80] H. Boschi-Filho, N. R. F. Braga and H. L. Carrion, Phys. Rev. D 73, 047901 (2006) arXiv:hep-th/0507063.

[81] N. Evans and A. Tedder, Phys. Lett. B 642, 546 (2006) arXiv:hep-ph/0609112.

[82] D. K. Hong, T. Inami and H. U. Yee, Phys. Lett. B 646, 165 (2007) arXiv:hepph/0609270].

[83] P. Colangelo, F. De Fazio, F. Jugeau and S. Nicotri, Phys. Lett. B 652, 73 (2007) arXiv:hep-ph/0703316].

[84] H. Forkel, Phys. Rev. D 78, 025001 (2008) arXiv:0711.1179 [hep-ph]].

[85] A. Vega and I. Schmidt, Phys. Rev. D 78, 017703 (2008) arXiv:0806.2267 [hep$\mathrm{ph}]$.

[86] K. Nawa, H. Suganuma and T. Kojo, Mod. Phys. Lett. A 23, 2364 (2008) arXiv:0806.3040 [hep-th].

[87] W. de Paula, T. Frederico, H. Forkel and M. Beyer, Phys. Rev. D 79, 075019 (2009) arXiv:0806.3830 [hep-ph]].

[88] P. Colangelo, F. De Fazio, F. Giannuzzi, F. Jugeau and S. Nicotri, Phys. Rev. D 78, 055009 (2008) arXiv:0807.1054 [hep-ph]].

[89] H. Forkel and E. Klempt, Phys. Lett. B 679, 77 (2009) arXiv:0810.2959 [hep$\mathrm{ph}]$. 
[90] H. C. Ahn, D. K. Hong, C. Park and S. Siwach, Phys. Rev. D 80, 054001 (2009) arXiv:0904.3731 [hep-ph]].

[91] Y. Q. Sui, Y. L. Wu, Z. F. Xie and Y. B. Yang, Phys. Rev. D 81, 014024 (2010) arXiv:0909.3887 [hep-ph]].

[92] J. I. Kapusta and T. Springer, Phys. Rev. D 81, 086009 (2010) arXiv:1001.4799 [hep-ph]];

[93] P. Zhang, Phys. Rev. D 81 (2010) 114029 [arXiv:1002.4352 [hep-ph]]; JHEP 1005 (2010) 039 arXiv:1003.0558 [hep-ph]]; arXiv:1007.2163 [hep-ph].

[94] See also: S. J. Wang, J. Tao, X. B. Guo and L. Li, arXiv:1007.2462 [hep-th].

[95] H. J. Kwee and R. F. Lebed, JHEP 0801, 027 (2008) arXiv:0708.4054 [hep-ph]].

[96] H. R. Grigoryan and A. V. Radyushkin, Phys. Rev. D 76, 115007 (2007) arXiv:0709.0500 [hep-ph]].

[97] C. A. B. Bayona, H. Boschi-Filho, M. Ihl and M. A. C. Torres, arXiv:1006.2363 [hep-th].

[98] S. J. Brodsky and G. F. de Teramond, arXiv:0802.0514 [hep-ph].

[99] G. F. de Teramond and S. J. Brodsky, Phys. Rev. Lett. 94, 201601 (2005) arXiv:hep-th/0501022.

[100] H. R. Grigoryan and A. V. Radyushkin, Phys. Rev. D 76, 095007 (2007) arXiv:0706.1543 [hep-ph]].

[101] The data compilation is from M. Diehl, Nucl. Phys. Proc. Suppl. 161, 49 (2006) arXiv:hep-ph/0510221.

[102] G. Grunberg, Phys. Lett. B 95, 70 (1980); Phys. Rev. D 29, 2315 (1984); Phys. Rev. D 40, 680 (1989).

[103] S. J. Brodsky and H. J. Lu, Phys. Rev. D 51, 3652 (1995) arXiv:hepph/9405218]; S. J. Brodsky, G. T. Gabadadze, A. L. Kataev and H. J. Lu, Phys. Lett. B 372, 133 (1996) arXiv:hep-ph/9512367.

[104] T. Appelquist, M. Dine and I. J. Muzinich, Phys. Lett. B 69, 231 (1977).

[105] J. D. Bjorken, Phys. Rev. 148, 1467 (1966).

[106] A. Deur, arXiv:0907.3385 [nucl-ex].

[107] S. J. Brodsky, G. F. de Teramond, A. Deur, Phys. Rev. D81, 096010 (2010) arXiv:1002.3948 [hep-ph]]. 
[108] J. M. Cornwall, Phys. Rev. D 26, 1453 (1982).

[109] S. D. Drell and A. C. Hearn, Phys. Rev. Lett. 16, 908 (1966); S. B. Gerasimov, Sov. J. Nucl. Phys. 2 (1966) 430 [Yad. Fiz. 2 (1966) 598].

[110] H. Gao and L. Zhu, AIP Conf. Proc. 747, 179 (2005) arXiv:nucl-ex/0411014.

[111] J. C. R. Bloch, Phys. Rev. D 66, 034032 (2002) arXiv:hep-ph/0202073; P. Maris and P. C. Tandy, Phys. Rev. C 60, 055214 (1999) |arXiv:nuclth/9905056|; C. S. Fischer and R. Alkofer, Phys. Lett. B 536, 177 (2002) |arXiv:hep-ph/0202202]; C. S. Fischer, R. Alkofer and H. Reinhardt, Phys. Rev. D 65, 094008 (2002) |arXiv:hep-ph/0202195|; R. Alkofer, C. S. Fischer and L. von Smekal, Acta Phys. Slov. 52, 191 (2002) [arXiv:hep-ph/0205125]; M. S. Bhagwat, M. A. Pichowsky, C. D. Roberts and P. C. Tandy, Phys. Rev. C 68, 015203 (2003) arXiv:nucl-th/0304003.

[112] V. D. Burkert and B. L. Ioffe, Phys. Lett. B 296, 223 (1992); J. Exp. Theor. Phys. 78, 619 (1994) [Zh. Eksp. Teor. Fiz. 105, 1153 (1994)].

[113] G. Parisi, Phys. Lett. B 39, 643 (1972).

[114] S. J. Brodsky, D. S. Hwang and I. Schmidt, Phys. Lett. B 530, 99 (2002) arXiv:hep-ph/0201296.

[115] J. C. Collins, Phys. Lett. B 536, 43 (2002) arXiv:hep-ph/0204004.

[116] D. Boer, S. J. Brodsky and D. S. Hwang, Phys. Rev. D 67, 054003 (2003) arXiv:hep-ph/0211110|.

[117] S. J. Brodsky, A. H. Hoang, J. H. Kuhn and T. Teubner, Phys. Lett. B 359, 355 (1995) arXiv:hep-ph/9508274.

[118] J. Collins and J. W. Qiu, Phys. Rev. D 75, 114014 (2007) arXiv:0705.2141 [hep-ph]]. 